\title{
Direct visualization of the Campbell regime in superconducting stripes
}

\author{
R. B. G. Kramer, G. W. Ataklti, V. V. Moshchalkov, and A. V. Silhanek \\ Institute for Nanoscale Physics and Chemistry (INPAC), Nanoscale Superconductivity and Magnetism Group, K.U.Leuven, \\ Celestijnenlaan 200D, B-3001 Leuven, Belgium
}

(Received 23 February 2010; published 19 April 2010)

\begin{abstract}
A combination of scanning Hall microscopy and scanning ac-susceptibility measurements in superconducting stripes (ribbons) of width $w<10 \mu \mathrm{m}$ was used to observe the dimensional phase transitions of the vortex lattice and its stability under alternating fields. At low dc magnetic fields applied perpendicularly to the plane of the stripes, vortices form a one-dimensional chain at the center of the stripes. Above a certain field $H^{*}(w)$, the vortex chain splits in two parallel rows displaced laterally in such a way that a zigzag vortex pattern is observed. By shaking the vortices with an external magnetic ac field and detecting their in-phase motion locally, we can identify the degree of mobility of each individual vortex. This technique allows us (i) to directly visualize the transition from intravalley (Campbell regime) to intervalley vortex motion as the amplitude of the ac modulation is increased and (ii) to accurately determine the temperature at which the vortex lattice freezes in a field-cooling experiment.
\end{abstract}

DOI: 10.1103/PhysRevB.81.144508

PACS number(s): 74.78.Na, 74.25.N-

The hallmark of type II superconductivity is the presence of quantized magnetic flux encircled by a rotating condensate of paired electrons when a sufficiently strong magnetic field is applied. The free motion of these fluxons leads to dissipation thus destroying the perfect conductivity of the system. The ever growing electricity demand has motivated for several decades a fierce strive for understanding, improving, and optimizing the mechanisms to prevent the fluxon's motion by introducing a rich diversity of pinning centers. ${ }^{1}$

Among the most powerful experimental methods used to determine the efficiency of pinning sites is the ac-susceptibility technique which consist of shaking the flux-line lattice with a small alternating magnetic field while recording its in-phase magnetic response. ${ }^{2}$ Pioneering theoretical works by Campbell and co-workers substantially contributed to comprehend and interpret the ac response of a superconducting system. ${ }^{3,4}$ In the most simple version, two different regimes with distinctive ac responses can be identified. For a gentle ac shacking, flux lines oscillate inside the pinning potential following a reversible and low dissipative motion (Campbell regime). By increasing the strength of the ac excitation, the intravalley vortex motion first senses the anharmonicities of the potential well and eventually switches to an intervalley motion where vortices can hop from one pinning site to another. ${ }^{5}$

So far, all the experimental and theoretical reports are focused on global ac-susceptibility investigations where the recorded signal represents an average over millions of flux lines each of which trapped in a different pinning potential and subjected to a different environment. ${ }^{6-8}$ In this paper, we introduce a local vortex imaging technique with singlevortex resolution which allows us to directly visualize the oscillatory motion of individual vortices submitted to harmonic ac excitations. The system to investigate consists of superconducting ribbons which, due to the proximity of the flux lines to the border, are particularly suitable for an efficient vortex shaking with an alternating external field. Scanning Hall microscopy characterization of the superconducting stripes done after field-cooling procedure shows that at low fields, vortices sit at the center of the stripes forming a one-dimensional chain. At higher fields, this chain becomes unstable due to the strong vortex-vortex interaction and it splits in two lines of vortices. By scanning ac-susceptibility microscopy (SSM), we demonstrate that, due to symmetry considerations, vortices located at the center of the stripe cannot be displaced by the external ac field. In contrast to that, off-center vortices which are either trapped by a pinning center or due to a zigzag vortex configuration, can be easily excited by the external ac field. We present direct observation of the transition from intravalley to intervalley vortex motion by performing SSM at different ac amplitudes. Furthermore, this technique also allows us to unambiguously determine the temperature at which the flux-line lattice is frozen in a typical field-cooling procedure.

The investigated samples consist of $\mathrm{Pb}$ ribbons $0.5 \mathrm{~mm}$ long, $50 \mathrm{~nm}$ thick, and width ranging from 4 to $9 \mu \mathrm{m}$ in steps of $1 \mu \mathrm{m}$. The patterning was performed by e-beam lithography onto a $\mathrm{SiO}_{2}$ substrate covered with a double resist layer. The $\mathrm{Pb}$ deposition was done via electronbeam evaporation at $10^{-8}$ Torr nominal pressure while cooling the substrate with liquid nitrogen. The resulting stripes exhibit a superconducting transition at $T_{c}=7.20 \mathrm{~K}$ with a residual resistivity $\rho_{0}=1.75 \mu \Omega \mathrm{cm}$. From the slope of the superconducting-normal phase boundary and $\rho_{0}$, we estimate, following Ref. 9, a superconducting coherence length at zero temperature $\xi(0) \sim 34 \mathrm{~nm}$ and penetration depth $\lambda(0) \sim 52 \mathrm{~nm}$.

In order to identify the field at which vortices first penetrate into the superconducting stripes and the different vortex configurations occurring at higher fields, we first acquired a series of scanning Hall-probe microscopy (SHPM) images using a modified low-temperature scanning Hallprobe microscope from Nanomagnetics Instruments. ${ }^{10}$ In all cases, a field-cooling procedure is made before scanning the Hall probe at a scan speed of $60 \mu \mathrm{m} / \mathrm{s}$. Figure 1 summarizes the different vortex configurations obtained at different fields and for several widths $w$ of the $\mathrm{Pb}$ ribbons. Two characteristic fields, $H_{p}$ and $H^{*}$, are indicated in this figure. For $H$ $<H_{p}$, the stripe is in the Meissner state as shown in Fig. 1(a) for the $5-\mu \mathrm{m}$-wide sample. At $H=H_{p}$, the first vortex enters 


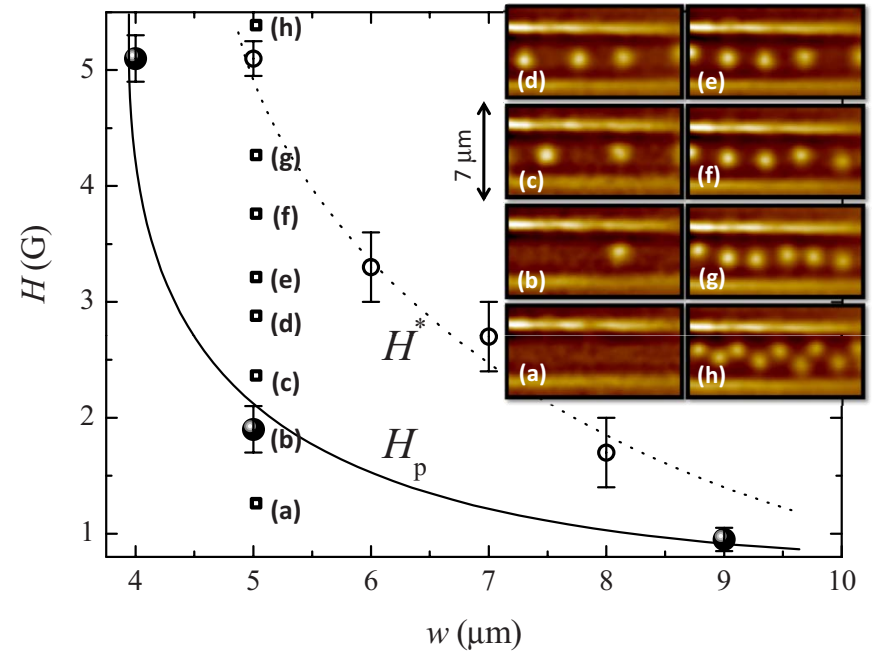

FIG. 1. (Color online) Main panel: transition field, $H_{p}$, from Meissner state to vortex penetration and from single-to-double-row vortex configuration, $H^{*}$, as a function of stripe width $w$. The lines are a guide to the eyes. The insets show scanning Hall-probe microscopy images in a $7 \times 14 \mu \mathrm{m}^{2}$ area for the 5 - $\mu \mathrm{m}$-wide stripe obtained at $4.2 \mathrm{~K}$ after field cooling. Insets (a)-(h) correspond to different fields as indicated by square open symbols in the main panel.

into the stripe [see Fig. 1(b) and the corresponding point in the main panel]. Further increasing the field leads to the penetration of additional vortices and consequently to a progressive compression of the vortex row [Figs. $1(\mathrm{c})-1(\mathrm{~g})]$. At $H$ $=H^{*}$, the vortex row undergoes a buckling transition and splits in two chains forming a zigzag pattern [Fig. 1(h)]. As expected, the wider the stripe the shallower the confining potential and therefore the smaller $H^{*}{ }^{11}$ Interestingly, further increasing the field, first an almost square array of vortices is observed $^{12}$ before a more disordered configuration is achieved. The field $H_{p}$ at which the first vortex penetrates in narrow stripes and the formation of different vortex patterns have been previously investigated both theoretically ${ }^{12-20}$ and experimentally. ${ }^{21-28}$ Similarly to early scanning Hall experiments performed by Stan et al. ${ }^{27}$ in $\mathrm{Nb}$ stripes, our determination of $H_{p}$ is about four times larger than the one predicted theoretically.

In order to investigate the stability of the different vortex configurations under small ac excitations, we used a technique ${ }^{29}$ consisting of picking up the local ac signal with the Hall cross at the same frequency than the oscillating field $h_{a c}$. Figure 2(a) shows the in-phase signal $\left(\chi^{\prime}\right)$ and the outof-phase signal $\left(\chi^{\prime \prime}\right)$ as a function of temperature $T$ when the Hall cross is situated at the center of a $7-\mu \mathrm{m}$-wide ribbon at $H=0 \mathrm{G}$, ac amplitude $h_{a c}=1 \mathrm{G}$ and frequency $f=77.5 \mathrm{~Hz}$. As in the standard ac-susceptibility technique, $\chi^{\prime}$ accounts for the ability of the system to screen out the oscillating field whereas $\chi^{\prime \prime}$ is related to the irreversibilities. For demonstration of the working principle, we performed SHPM [Figs. 2(b) and 2(c)] and SSM [Fig. 2(d)] at the extreme end of a 9- $\mu \mathrm{m}$-wide $\mathrm{Pb}$ stripe after field cooling at $H=1.3 \mathrm{G}$ down to $T=7 \mathrm{~K}$.

The two circles in Figs. 2(b) and 2(c) indicate the position of the vortices after field cooling. Figure 2(b) was then ob-

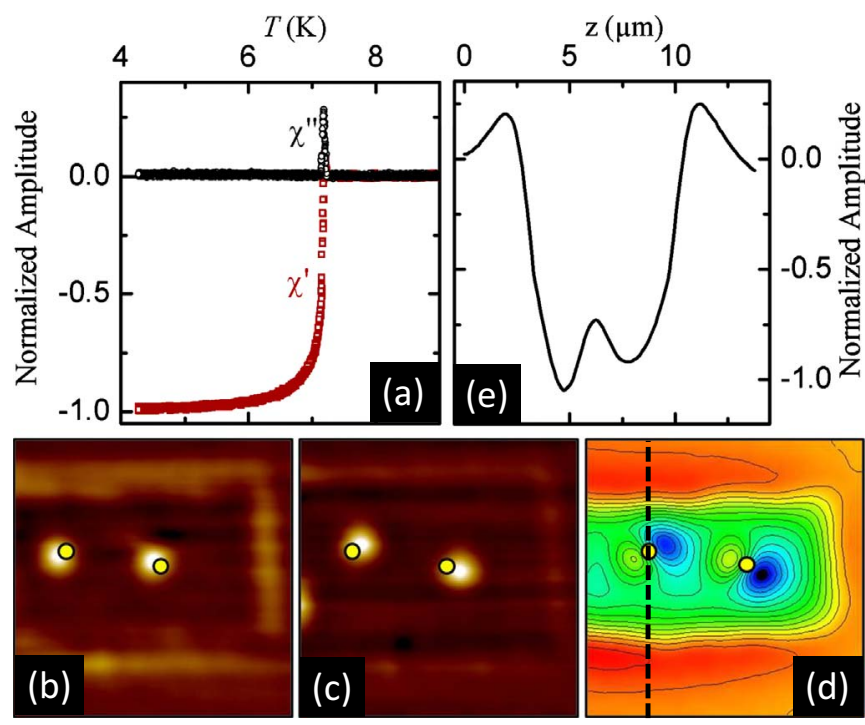

FIG. 2. (Color online) (a) In-phase $\left(\chi^{\prime}\right)$ and out-of-phase $\left(\chi^{\prime \prime}\right)$ ac signal picked up by the Hall cross located at the center of a $7 \mu \mathrm{m}$ stripe, using an ac amplitude of $1 \mathrm{G}$ and a frequency of $f$ $=77.5 \mathrm{~Hz}$. (b) and (c) Scanning Hall-probe images of a 9- $\mu \mathrm{m}$-wide stripe field cooled at $H=1.3 \mathrm{G}$ to $7 \mathrm{~K}$ and then applying $2.3 \mathrm{G}$ and $0.3 \mathrm{G}$, respectively. (d) Scanning ac-susceptibility image acquired at $7 \mathrm{~K}$ with ac amplitude $1 \mathrm{G}$ and frequency $f=77.5 \mathrm{~Hz}$. Dots in (b)-(d) indicate the average vortex position. The image size is $14 \times 14 \mu \mathrm{m}^{2}$. (e) ac-susceptibility line profile along the dashed line in panel $(d)$.

tained at the maximum of a $h_{a c}=1 \mathrm{G}$ ac oscillation, i.e., $H$ $+h_{a c} \sim 2.3 \mathrm{G}$ whereas Fig. 2(c) was recorded at the minimum of the oscillation $H-h_{a c} \sim 0.3 \mathrm{G}$. The relative displacement of the vortices with respect to the equilibrium position becomes apparent. The scanning ac-susceptibility microscopy image obtained under the same ac excitation [Fig. 2(d)] shows that the resulting periodic oscillation of these vortices gives rise to two distinct spots corresponding to positive (yellowish or brighter) and negative (blue or darker) pickup ac signal. The physical origin of these two spots can be directly correlated with the images in Figs. 2(a) and 2(b). When the external field is increased, the screening currents push the vortices toward the center of the sample and consequently the Hall signal increases when scanning in those areas where vortices move into. At the same time, i.e., while $h_{a c}(t)$ is increasing, the signal decreases near the border of the sample. This decrease in the signal is $180^{\circ}$ out-ofphase with respect to the excitation and therefore appears as a dark spot. The SSM signal not only detects the motion of vortices but also the oscillation of the screening current next to the sample border. This is clearly seen in Fig. 2(e) where a cross section of the SSM image is shown along the dotted black line indicated in Fig. 2(d). Notice that the amplitude of the susceptibility vs position exhibits two peaks at the samples' borders. These peaks are surprising since they seem to indicate a negative screening. However, it is easy to show that they arise from an amplification of the fields at the samples' borders due to demagnetization effects.

In Fig. 3, we illustrate further the power of this technique for visualizing the vortex mobility at different vortex con- 


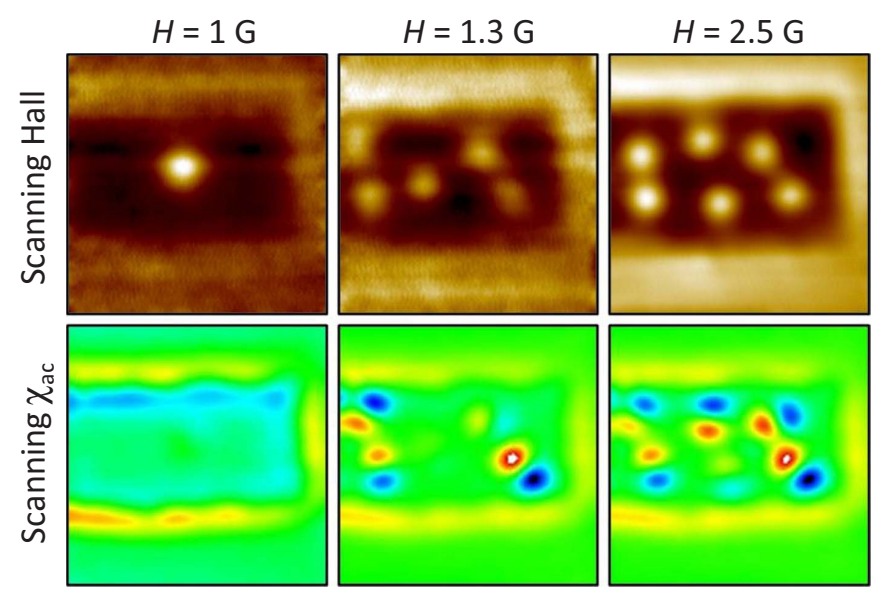

FIG. 3. (Color online) Scanning Hall-probe microscopy (upper row) and simultaneously obtained scanning ac-susceptibility microscopy (bottom row) images for three different fields (different columns) for the $9-\mu \mathrm{m}$-wide stripe at $T=7 \mathrm{~K}$.

figurations, i.e., dc fields, for the $9 \mu \mathrm{m}$ stripe at $4.2 \mathrm{~K}$. The upper row shows the SHPM images whereas the bottom row shows the simultaneously acquired SSM pictures. In order to stress the contrast due to the vortex motion, in the SSM images we have subtracted the smooth background produced by the screening currents. The leftmost column of Fig. 3, obtained at $H=1 \mathrm{G}$, shows a single vortex located at the equator of the stripe. This very symmetric position makes that the force produced by the Meissner currents running at the upper edge of the stripe cancel out the force due to the supercurrents at the lower edge of the stripe, thus leading to an almost undetectable ac signal in the SSM image. In this case, the symmetry is only broken by the current bending at the end of the stripe which induces a vortex displacement mainly in the east-west direction.

The middle column in Fig. 3 shows a five-vortex pattern obtained after field cooling at $H=1.3 \mathrm{G}$ forming an inverted $\mathrm{N}$-shape structure which appears as a precursor of the zigzag pattern. Interestingly, only four out of five vortices seem to move as a response to the external oscillatory field. This lack of motion of the central vortex can be understood as a result of a highly symmetric environment of both, the Meissner screening currents and the surrounding vortices. The rightmost column of Fig. 3 shows a disordered vortex zigzag pattern obtained at $H=2.5 \mathrm{G}$. The most obvious feature of this figure is the alternating vortex lattice compression and decompression in the direction perpendicular to the sample's borders as a result of the oscillating ac field. Notice that both the fully compressed and decompressed lattices still retain the zigzag structure, pointing out to the robust stability of this particular vortex distribution. This is in agreement with the wide range of field where this particular vortex pattern is observed.

As already pointed out by Campbell, vortex shaking inside the pinning potential well can only occur for moderate ac amplitudes $h_{a c}$. In other words, beyond a certain oscillation amplitude, vortices can make excursions out of the pinning range. The most distinctive feature of this transition from intravalley to intervalley vortex motion is that the low-

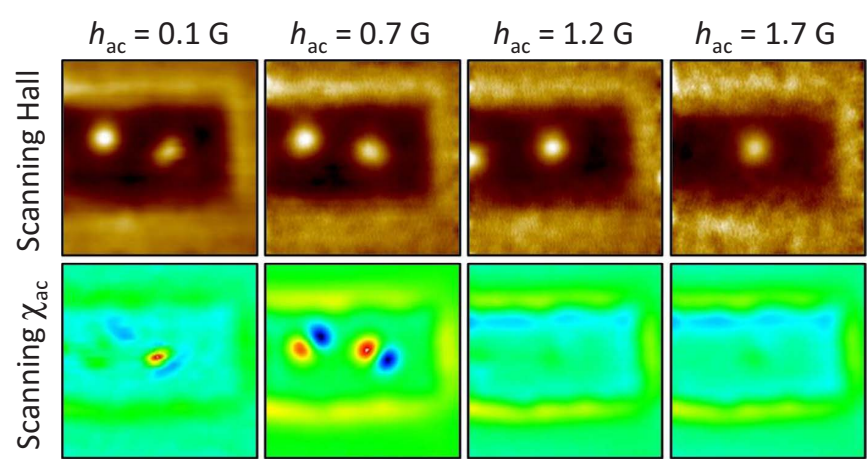

FIG. 4. (Color online) Vortex oscillation as a function of the ac amplitude $h_{a c}$. The upper row shows the scanning Hall microscopy images obtained at the end of a $9 \mu \mathrm{m}$ stripe, at $T=7 \mathrm{~K}$ and $H=1.3 \mathrm{G}$. The lower row shows the simultaneously acquired scanning ac-susceptibility microscopy images. Different columns correspond to different $h_{a c}$ always at a frequency $f=77.5 \mathrm{~Hz}$.

amplitude phase retains the original vortex configuration whereas in the latter, the initial vortex distribution can be altered. In order to observe this transition with single-vortex resolution, we performed a series of SHPM and SSM in the $9 \mu \mathrm{m}$ sample at fixed temperature $T=7 \mathrm{~K}$ and field $H$ $=1.3 \mathrm{G}$ while sweeping the ac amplitude $h_{a c}$ from 0 to $2 \mathrm{G}$ in steps of $0.1 \mathrm{G}$. A summary of the results is shown in Fig. 4 where the upper row shows the SHPM images and the lower row shows the SSM frames. It is worth mentioning that the initial vortex distribution is obtained via a fieldcooling process thus forcing the vortices to nucleate at the pinning sites. At low amplitudes (first column), a very weak SSM contrast evidences the small amplitude of oscillation of the vortex motion inside the pinning potential. For $h_{a c}$ $=0.7 \mathrm{G}$ (second column), the oscillatory motion of the two vortices, toward the center of the sample and perpendicular to the end of the stripe, is apparent. This oscillatory motion around the pinning potential corresponds to the Campbell regime. At $h_{a c}=1.2 \mathrm{G}$ (third column), a sudden loss of contrast in the SSM image results from the rearrangement of the vortex distribution to a more symmetric state. This transition which involves the depinning of vortices can be regarded as a local dynamically induced vortex ordering. For even higher ac amplitudes (fourth column), it is even possible to remove one of the vortices away from the sample.

It is worth noticing that in order to be able to obtain a detectable SSM signal, the scanning has to be carried out at high temperatures where pinning is relatively weak. This is at odds with the standard SHPM for which the contrast is highly improved as temperature is lowered. The question now arises at which temperature the vortex lattice is frozen in a field-cooling experiment? This is a relevant question for several techniques where field-cooling processes are typically used such as Bitter decoration or scanning Hall-probe microscopy. ${ }^{10,30}$ In order to address this issue, we investigated the vortex mobility via SSM in the $9 \mu \mathrm{m}$ sample, at $H=1.3 \mathrm{G}$ using $h_{a c}=1 \mathrm{G}$ while decreasing temperature in steps of $0.1 \mathrm{~K}$. The main results are shown in Fig. 5 where panel (a) shows the SHPM image after field cooling at $4.2 \mathrm{~K}$ indicating the frozen vortex pattern, and panels (b)-(d) show SSM images for different temperatures. At $T=7 \mathrm{~K}$, both vor- 

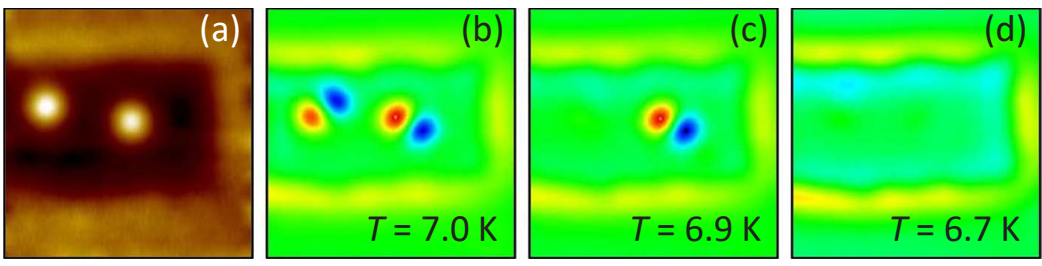

FIG. 5. (Color online) Scanning Hall-probe microscopy image of a 9 - $\mu \mathrm{m}$-wide stripe obtained at $4.2 \mathrm{~K}$ and $H=1.3 \mathrm{G}$, after field cooling (a). Panels (b)-(d) show scanning acsusceptibility microscopy images for the same vortex configuration than (a), at three different temperatures.

tices follow the ac excitation. This situation changes at $T$ $=6.9 \mathrm{~K}$ where one of the vortices is able to oscillate inside the pinning potential whereas the other remains nearly still. At $T=6.7 \mathrm{~K}$, two weak spots are indicative of very small vortex mobility. From this panels, we can unambiguously determine that the freezing temperature is about $6.8 \mathrm{~K}$ in the case of an ac excitation $h_{a c}=1$ G. As expected, lower amplitudes lead to higher freezing temperatures.

It is important to mention that attempts to measure the dependence of the vortex oscillation amplitude with $h_{a c}$ show almost no dependence but rather a change in the contrast as shown in Fig. 4. This might indicate that the size of the pinning center is much smaller than the superconducting coherence length.

In summary, we have introduced a technique, scanning ac-susceptibility microscopy, which permitted us to directly image the oscillatory motion of individual vortices under an external ac-field modulation. We show clear evidence that the transition from intravalley vortex motion (Campbell regime) to intervalley vortex motion passes through a dynamic ordering of the vortex lattice. Furthermore, we show that in a field-cooling experiment, the vortex lattice freezes at temperature very close to the critical temperature. This temperature could be determined accurately for $1 \mathrm{G}$ modulation field. Despite the fact that superconducting stripes have been studied intensively during the last decades, we provide different insights in the vortex patterns formation and their stability. The ac-susceptibility microscopy technique has been demonstrated to be a very powerful and promising tool that could be used, for instance, to directly visualize the length scale where ac fields penetrate in a superconducting sample (Campbell penetration depth, critical state penetration depth, etc.)

This work was supported by Methusalem Funding of the Flemish government, FWO-Vlaanderen, and the Belgian Inter-University Attraction Poles IAP Programmes. A.V.S. is grateful for the support from the FWO-Vlaanderen. The authors would like to thank Werner Gillijns for the critical reading of the manuscript.
${ }^{1}$ B. Maiorov, S. A. Baily, H. Zhou, O. Ugurlu, J. A. Kennison, P. C. Dowden, T. G. Hoelesinger, S. R. Foltyn, and L. Civale, Nature Mater. 8, 398 (2009).

${ }^{2}$ F. Gömöry, Supercond. Sci. Technol. 10, 523 (1997).

${ }^{3}$ A. M. Campbell, J. Phys. C 4, 3186 (1971).

${ }^{4}$ A. M. Campbell and J. E. Evetts, Adv. Phys. 21, 199 (1972).

${ }^{5}$ C. J. van der Beek, V. B. Geshkenbein, and V. M. Vinokur, Phys. Rev. B 48, 3393 (1993).

${ }^{6}$ G. Pasquini, P. Levy, L. Civale, G. Nieva, and H. Lanza, Physica C 274, 165 (1997); S. Raedts, A. V. Silhanek, V. V. Moshchalkov, J. Moonens, and L. H. A. Leunissen, Phys. Rev. B 73, 174514 (2006).

${ }^{7}$ J. R. Clem and A. Sanchez, Phys. Rev. B 50, 9355 (1994).

${ }^{8}$ E. H. Brandt, Phys. Rev. B 49, 9024 (1994).

${ }^{9}$ P. H. Kes and C. C. Tsuei, Phys. Rev. B 28, 5126 (1983).

${ }^{10}$ S. J. Bending, Adv. Phys. 48, 449 (1999).

${ }^{11}$ A. J. Drew, M. W. Wisemayer, D. O. G. Heron, S. Lister, S. L. Lee, A. Potenza, C. H. Marrows, R. M. Dalgliesh, T. R. Charlton, and S. Langridge, Phys. Rev. B 80, 134510 (2009).

${ }^{12}$ D. A. Luzhbin, Phys. Solid State 43, 1823 (2001).

${ }^{13}$ C. Bolech, G. C. Buscaglia, and A. Lopez, Phys. Rev. B 52, R15719 (1995).

${ }^{14}$ G. Carneiro, Phys. Rev. B 57, 6077 (1998).

${ }^{15}$ I. L. Maksimov and G. M. Maksimova, Pis'ma Zh. Eksp. Teor. Fiz. 65, 405 (1997).

${ }^{16}$ G. M. Maksimova, Phys. Solid State 40, 1607 (1998).

${ }^{17}$ J. J. Palacios, Phys. Rev. B 57, 10873 (1998).
${ }^{18}$ E. Sardella, M. M. Doria, and P. R. S. Netto, Phys. Rev. B 60, 13158 (1999).

${ }^{19}$ C. C. de Souza and J. A. Aguiar, Physica C 388-389, 673 (2003).

${ }^{20}$ E. Bronson, M. P. Gelfand, and S. B. Field, Phys. Rev. B 73, 144501 (2006).

${ }^{21}$ J. Guimpel, L. Civale, F. de la Cruz, J. M. Murduck, and I. K. Schuller, Phys. Rev. B 38, 2342 (1988).

${ }^{22}$ S. H. Brongersma, E. Verweij, N. J. Koeman, D. G. de Groot, R. Griessen, and B. I. Ivlev, Phys. Rev. Lett. 71, 2319 (1993).

${ }^{23}$ G. Karapetrov, J. Fedor, M. Iavarone, D. Rosenmann, and W. K. Kwok, Phys. Rev. Lett. 95, 167002 (2005).

${ }^{24}$ A. Pan, M. Ziese, R. Hohne, P. Esquinazi, S. Knappe, and H. Koch, Physica C 301, 72 (1998).

${ }^{25}$ U. Patel, S. Avci, Z. L. Xiao, J. Hua, S. H. Yu, Y. Ito, R. Divan, L. E. Ocola, C. Zheng, H. Claus, J. Hiller, U. Welp, D. J. Miller, and W. K. Kwok, Appl. Phys. Lett. 91, 162508 (2007).

${ }^{26}$ B. L. T. Plourde, D. J. Van Harlingen, R. Besseling, M. B. S. Hesselberth, and P. H. Kes, Physica C 341-348, 1023 (2000).

${ }^{27}$ G. Stan, S. B. Field, and J. M. Martinis, Phys. Rev. Lett. 92, 097003 (2004).

${ }^{28}$ M. Ziese, P. Esquinazi, P. Wagner, H. Adrian, S. H. Brongersma, and R. Griessen, Phys. Rev. B 53, 8658 (1996).

${ }^{29}$ M. P. DeFeo and M. Marchevsky, Phys. Rev. B 73, 184409 (2006).

${ }^{30}$ Y. Fasano and M. Menghini, Supercond. Sci. Technol. 21, 023001 (2008). 